\title{
Research on New Intelligent Industrial Control and Communication System based on PLC Technique
}

\author{
LiHong Deng ${ }^{1}$ \\ ${ }^{1}$ xi'an Aeronautical University , \\ xi'an city,Shaanxi Province, China
}

\begin{abstract}
With the progress of computer technology and intelligent control system, it is necessary to introduce the PLC together with the current systems. In this paper, we conduct research on the new intelligent industrial control and communication system based on PLC technique. As Industry 4.0 at the core of intelligent factory, the products made in its basic idea is integrated with dynamic digital memory, perception and communication ability, carrying the whole supply chain and all kinds of information required for life cycle. PLC biggest characteristic is to constitute the traditional relay model computer software technology, formed a unique style in relay ladder graph based image programming language, the PLC control system in the form of program to achieve its control function. In the simulation section, we implement our proposed system in detail and conduct numerical analtsis and simulation. The result is acceptable and accurate.
\end{abstract}

Keywords: Intelligent Industrial; Control and Communication; PLC Technique; System Building.

\section{Introduction}

In order to meet the quest for automation and flexibility, many complex manufacturing systems are controlled by Programmable Logical Controllers (PLC). Modern industrial control of PLC and touch screen control system is widely used, but the cost of PLC is higher, compared with the single chip microcomputer has obvious advantages on economy. With most of the PLC touch screen in support as well as support the MODBUS protocol, which makes the touch screen and single-chip microcomputer control system, this system has simple structure, stable performance, low cost, suitable for some small amount of information of industrial control system. Along with the computer technology, communication technology, control technology and graphics display technology, the development of industrial control system (distributed control system, fieldbus control system, etc.) at home and abroad, obtained the rapid development and wide application. With the increasing scale of control system in the treatment of network applications, on the one hand, in the development and configure the network involved in the program "intrinsic" and "accidental" complexity problem of the network application is often more difficult to design, implementation and debugging, in terms of efficiency and stability is difficult to guarantee; Software reusability, on the other hand, has been widely followed, and less consider the design results of industrial control system design reusability, once the design change, can lead to network application change sharply, waste of resources, and influence on the reliability of the system. PLC control system is generally used for analog control, position control, etc. PLC biggest characteristic is to constitute the traditional relay model computer software technology, formed a unique style in relay ladder graph based image programming language, the PLC control system in the form of program to achieve its control function. Therefore, PLC programming technique in PLC control system has a very important position.

As a condition of talents cultivation of hardware training equipment and the upgrading of advancing with the times that is particularly important. Aimed at this situation, the school should adapt to social development needs, 
develop the corresponding professional and technical personnel, not only to let them understand and master the relevant professional knowledge, and have strong ability of problem analysis and solving ability. With the rapid development of economic construction, many of the spatial grid steel structure of large construction all. Because this structure is built by welding or bolt connection of various kinds of nodes, the different shape of steel bar (or components) of the entity, with sufficient strength and rigidity, it is easy to do big span, is newly built large railway station, optimization of sports venues, conference and exhibition center, etc. As Industry 4.0 at the core of intelligent factory, the products made in its basic idea is integrated with dynamic digital memory, perception and communication ability, carrying the whole supply chain and all kinds of information required for life cycle; The whole value chain integrated production facilities can be achieved by the organization, and the flexibility to decide according to the present situation of the production process. Smart factory's goal is to establish a personalized and highly flexible production mode of digital products and services. Therefore, we conduct corresponding research on the new intelligent industrial control and communication system based on PLC technique in this paper. In the following sections, we will discuss the technique in detail.

\section{The Proposed Technique}

The Intelligent Industrial Control. Pressure and flow rate is an important physical parameter in variable spray control system, and the general variable spray control system are greatly influenced by the environment, equipment error, and has the characteristics of nonlinear, time-varying, big lag. Conventional PID control to adapted to the ideal linear control system, for the characteristics of nonlinear, time-varying, big lag and so on the characteristic of the control system could not meet the requirement of the control, to reach the ideal control effect. So for this defect, this paper proposes a fuzzy self-tuning PID control based on PLC control variables of the spray control system. Variable selection control system of electric spray PI regulator for the controlled object, of the opening of the regulating valve is adjustable, and the size of the spray quantity control system. According to the control system of variable spray pressure, the flow sensor feedback data and control the size of the electric control valve opening. Electric control valve itself is a kind of complex control system, can be as simple as a second order system with time delay shown in the formula 1 .

$$
G(s)=\frac{K \exp (-\tau s)}{\left(T_{1} s+1\right)+\left(T_{2} s+1\right)+\ldots+\left(T_{n} s+1\right)}
$$

PLC control of fuzzy self-tuning PID control combined with PLC control is flexible and changeable and adaptive fuzzy control, through the mathematical modeling of the control system of variable spray, by electric PI regulator is established as the core of fuzzy self-tuning PID control system. In the figure 1, we show the system description for the technique.

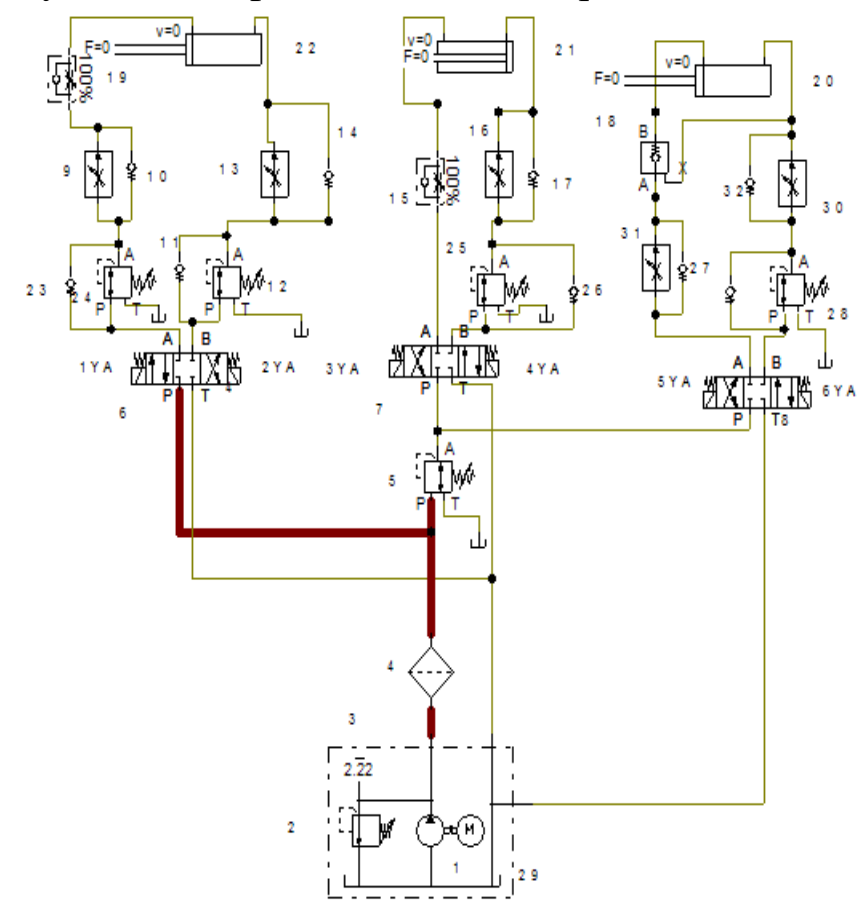

Fig. 1The Detailed Structure of the Control System 
Magnitude at all points of large-scale systems, applications, these nodes can be divided into one or more control domain and operation domain, each operation domain can monitor multiple control field and at the same time for joint monitoring and control of the domain. System and network related more operations, data encapsulation in objectification interface, and separation in multiplex framework to improve the reuse of the middleware, such as using the session redundancy and heartbeat detection mechanisms such as improve the tolerance of the network communication, real-time performance.

The Principles of Programmable Logical Controllers. In order to meet the general technological requirements of the controlled system, thus improve the production efficiency and product quality, the PLC control system design should follow the following basic principles. (1) Can satisfy the requirement of the controlled object control. The first premise of PLC control system design is to give full play to the function of PLC, maximum limit satisfy the requirement of the controlled object control. (2) PLC control system to ensure long-term safe, reliable and stable operation. In system design, component selection, software programming and so on comprehensive consideration, makes every effort to control the system safe and reliable. (3) To meet the design of control system is simple, economic and convenient use and maintenance. (4) Meet the needs of the development. To meet these principles should be on the basis of fully considering the need of the development of production and process improvement in the future. With PLC in industrial control and the popularization, more and more kinds of PLC products, the structure model, performance, capacity, instruction system, programming method, and so on each are not identical, applicable occasions also have focus. Therefore, reasonable selection of PLC, to improve the application of PLC in the control system has an important role. In the process of the PLC control system design, if some problem processes improper, will affect the normal running of the whole control system, which affect the enterprise's safety production and economic benefits. In order to improve the system reliability, ensure safe and efficient industrial facilities, in the process of the design of the system, we should pay attention to the following questions. (1) Make the output module (interface) load with certain margin. Output module is the most vulnerable to damage the parts PLC device itself. The simplest method is to reduce the output interface load adding power amplifier to the link, which USES suction power and keeping power is relatively small, small intermediate relay. (2) Pay attention to the output module of open circuit protection. In order to prevent due to reasons such as external short circuit output interface damage, short circuit protection device can be installed at the output interface. (3) Hardware setup of interlocking, interlocking function. Simple in PLC interlock on the internal logic and interlock, often in open circuit failure occurs. Such as motor positive and reverse contactor of interlock and interlock of ac, dc contactor, only in the application implementation is not enough. Because will often present main contactor contact "burn" phenomenon, the coil power after continue to open circuit fault, at this moment, such as giving the opposite control command can cause serious short circuit of main circuit. (4) The PLC power supply requirements and loss of pressure protection system. In large industrial and mining enterprises, due to the large electric equipment is more, the power supply quality is generally poor, interference, volatility, low voltage operation and instantaneous high pressure often appear, will have an effect on operation of PLC device. Although most of the PLC system has the strong power ability to adapt, but using high quality stabilized voltage supply will no doubt increase the reliability of the system. In application development, pay special attention to the system pressure loss protection, want to consider a state when the system pressure loss of the initial state recovery and interlock. (5) For requiring high reliability or power interference especially serious environment, can 
install 1 belt shielding layer of isolation transformer of variable ratio of $1: 1$, to reduce the interference between the device and ground. (6) PLC system security considerations. In order to ensure that the whole system can work in safe condition and reliable, to avoid failure due to the external power source, PLC is abnormal, wrong operation, and output error caused great economic losses and casualties, external PLC system should be installed the necessary protection circuit, such as acute blackout road, the power of overload protection, major fault alarm and protection, etc. In order to prevent the accident of power influence equipment operation, general PLC power supply will be used UPS power supply, UPS selection depends on the PLC with the load.

Output module according to the different way and relay output, the output transistor output and bidirectional three. To the perceptual load switch frequency, low power factor, can use the output

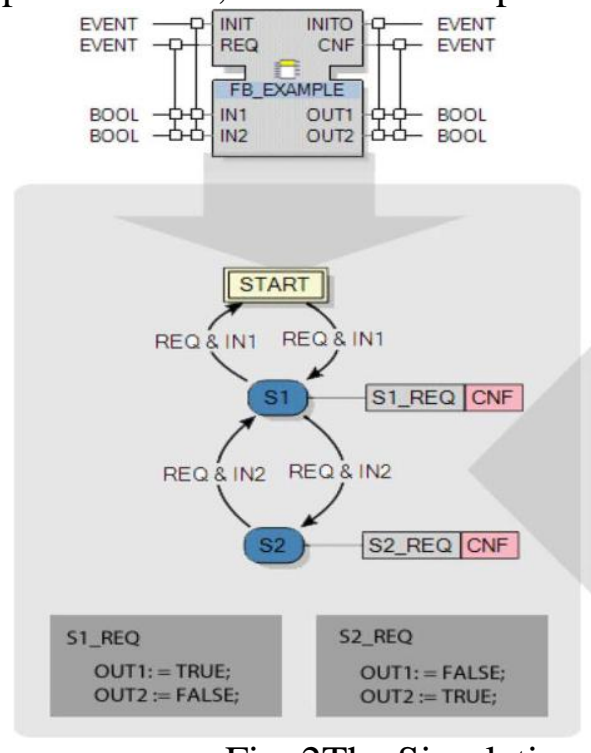

Fig. 2The Simulation for the Proposed System (ac output) or the transistor output (dc output), but this kind of module overload ability is a bit poor, the price also is higher. The ability to withstand overvoltage and overcurrent relay output module is stronger, price cheaper. Defect is slower response, the changes in the output is not soon, very frequent, can be used to give priority to. The corresponding simulation is shown in the next section.

\section{Experiment and Simulation}

The manipulator's upward swinging and downward swinging movements are controlled by electromagnet 1 YA, 2 YA respectively. The manipulator's clamping and loosening movements are controlled by electromagnet $5 \mathrm{YA}$, 6YA respectively. In addition, the systematic description of the novel proposed system is shown in the following figure.

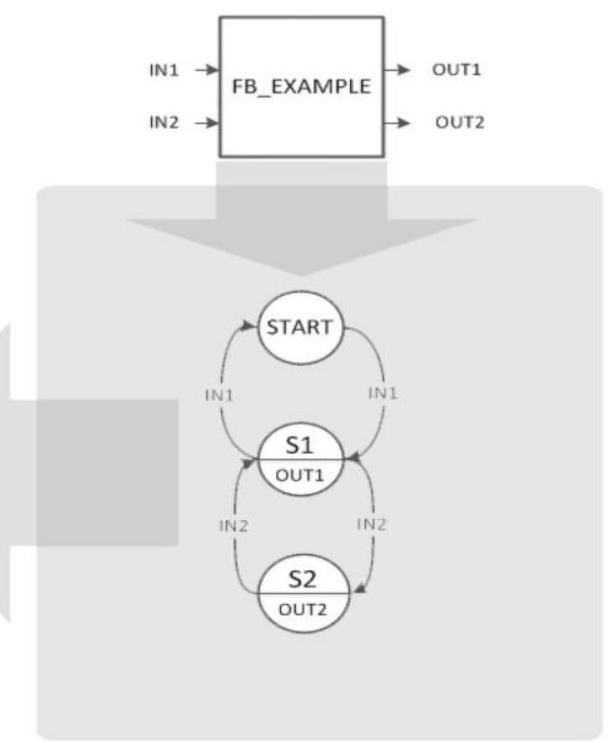

\section{CONCLUSIONS}

This paper provides a research on the new intelligent industrial control and communication system based on PLC technique. PLC control system is generally used for analog control, position control, etc. PLC biggest characteristic is to constitute the traditional relay model computer software technology, formed a unique style in relay ladder graph based image programming language, the PLC control system in the form of program to achieve its control function. Therefore, combining PLC with intelligent control system is urgent and necessary. In the future, we plan to conduct more related research on the control part to optimize the current system. 


\section{References}

[1] Xing Y. Transformation of Auxiliary Slope Hoist Adjust Speed of Electric Control System Based on PLC[J]. Coal Mine Machinery, 2014.

[2] Ming F, Gong-lei J, Hong-yong L, et al. Design of Automatic Control System for Chemical Production of Electronic Silver Powder[J]. Instrument Technique \& Sensor, 2014.
[3] Liu W, Bumiller G, Gao H. On (power-) line defined PLC system[J]. IEEE International Symposium on Power Line Communications \& Its Applications, 2014, 9074(8):81 - 86.

[4] Bao J, Wu H, Yan Y. A fault diagnosis system-PLC design for system reliability improvement[J]. International Journal of Advanced Manufacturing Technology, 2014, 75(1-4):523-534. 